\title{
Republic of Moldova: Statistical Appendix
}

This Statistical Appendix paper for the Republic of Moldova was prepared by a staff team of the International Monetary Fund as background documentation for the periodic consultation with the member country. It is based on the information available at the time it was completed on January 18, 2005. The views expressed in this document are those of the staff team and do not necessarily reflect the views of the government of the Republic of Moldova or the Executive Board of the IMF.

The policy of publication of staff reports and other documents by the IMF allows for the deletion of market-sensitive information.

To assist the IMF in evaluating the publication policy, reader comments are invited and may be sent by e-mail to publicationpolicy@imf.org.

\author{
Copies of this report are available to the public from \\ International Monetary Fund • Publication Services \\ 700 19th Street, N.W. • Washington, D.C. 20431 \\ Telephone: (202) 6237430 • Telefax: (202) 6237201 \\ E-mail: publications@imf.org • Internet: http://www.imf.org \\ Price: $\$ 15.00$ a copy

\section{International Monetary Fund \\ Washington, D.C.}





\title{
INTERNATIONAL MONETARY FUND
}

\author{
REPUBLIC OF MOLDOVA
}

\section{Statistical Appendix}

\author{
Prepared by Milan Cuc and Erik Lundback (EUR) \\ Approved by the European Department
}

January 18,2005

Contents

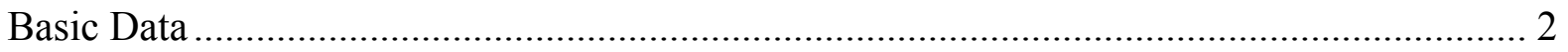

1. Gross Domestic Product by Expenditure 1998-2004 ………………………................. 3

2. Gross Domestic Product by Sector, 1998-2004 …….............................................. 4

3. Industrial Production by Industry, 1998-2004 ……................................................. 5

4. Agricultural and Industrial Production Indices, 1998-2004 ....................................... 7

5. Structure of Agricultural Land Ownership, 1994-2004 ……………………................ 8

6. Agricultural Production by Sector, 1998-2004 …….................................................. 9

7. Agricultural Productivity of Public Versus Private Sector, 1998-2003 ........................ 10

8. Nominal Wages by Sector, 1998-2004 ................................................................ 11

9. Employment by Sector, 1998-2003 ......................................................................... 12

10. Unemployment, Unpaid Leave, and Part-time Employment, 1998-2004 .................... 13

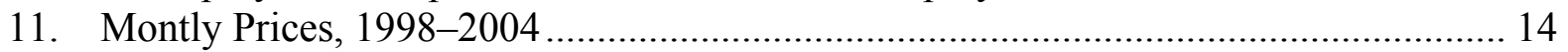

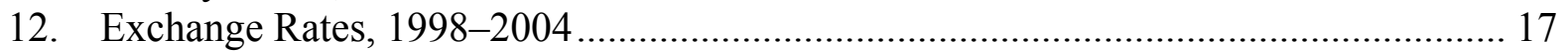

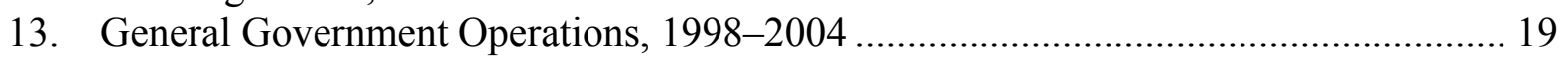

14. General Government Operations, 1998-2004 ………............................................. 20

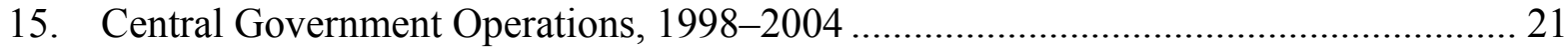

16. Local Government Operations, 1998-2004 _......................................................... 22

17. Central Government Expenditures by Main Budget Categories, 1998-2003 ................. 23

18. Local Government Expenditures by Main Budget Categories, 1998-2003 ................... 24

19. Accounts of the National Bank of Moldova, 1998-2004 ………................................ 25

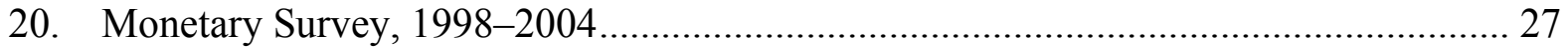

21. Balance of Payments, 1998-2004 ………………..................................................... 29

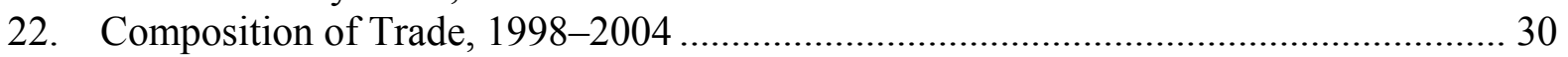

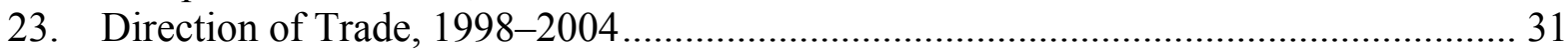

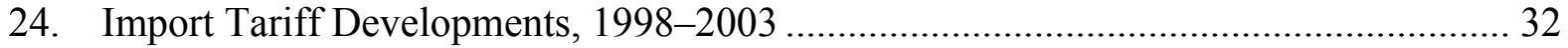

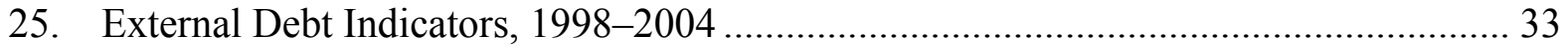




\section{Moldova: Basic Data 1/}

\begin{tabular}{lrr}
\hline & & \\
Social and demographic indicators & & \\
Area (including Transnistria) & 33,846 & sq. km. \\
Population & 3.6 & million \\
Share of urban population & 41 & percent \\
Rate of population growth & -0.3 & percent \\
Male life expectancy at birth & 64 & years \\
Female life expectancy at birth & 72 & years \\
Infant mortality rate (per 1,000 births) & 14.4 & \\
Hospital beds (per 10,000 people) & 68 & \\
Primary education (1-4 year) enrolment rate & 95 & percent \\
Secondary education (5-9 year) enrolment rate & 88 & percent \\
Gini-coefficient & 0.4 & \\
\hline
\end{tabular}

Sources: Moldovan Department for Statistics and Sociology and Fund staff estimates.

1/ Excluding Transnistria (except otherwise indicated). 
Table 1. Moldova: Gross Domestic Product by Expenditure 1998-2004 1/ (In millions of lei; at current prices)

\begin{tabular}{|c|c|c|c|c|c|c|c|}
\hline & 1998 & 1999 & 2000 & 2001 & 2002 & 2003 & $2004 \mathrm{H} 1$ \\
\hline Gross domestic product, official & 9,122 & 12,322 & 16,020 & 19,052 & 22,556 & 27,297 & 12,995 \\
\hline Final consumption expenditure & 9,203 & 11,090 & 16,503 & 19,263 & 23,289 & 29,706 & 14,406 \\
\hline Private & 6,876 & 9,137 & 14,031 & 16,385 & 18,493 & 24,417 & 11,756 \\
\hline Public & 2,327 & 1,953 & 2,472 & 2,878 & 4,796 & 5,289 & 2,650 \\
\hline Gross capital formation & 2,360 & 2,820 & 3,836 & 4,436 & 4,886 & 5,916 & 2,676 \\
\hline Fixed capital & 2,011 & 2,272 & 2,472 & 3,190 & 3,682 & 4,668 & 2,413 \\
\hline Stockbuilding & 349 & 548 & 1,364 & 1,246 & 1,204 & 1,248 & 263 \\
\hline Net exports & $-2,441$ & $-1,588$ & $-4,319$ & $-4,647$ & $-5,619$ & $-8,325$ & $-4,087$ \\
\hline \multicolumn{8}{|l|}{ Memorandum items: } \\
\hline Real GDP & -6.5 & -3.4 & 2.1 & 6.1 & 7.8 & 6.3 & 6.5 \\
\hline Implicit GDP deflator & 9.5 & 39.8 & 27.3 & 12.1 & 9.8 & 13.9 & 10.0 \\
\hline
\end{tabular}

Sources: Moldovan Department for Statistics and Sociology and Fund staff estimates.

1/ Excludes Transnistria. 
Table 2. Moldova: Gross Domestic Product by Sector, 1998-2004

(In millions of lei at current prices)

\begin{tabular}{|c|c|c|c|c|c|c|c|}
\hline & 1998 & 1999 & 2000 & 2001 & 2002 & 2003 & $2004 \mathrm{H} 1$ \\
\hline Value added & 7,719 & 10,999 & 14,022 & 16,774 & 19,689 & 23,356 & 11,047 \\
\hline Agriculture and fishing & 2,351 & 3,066 & 4,070 & 4,272 & 4,742 & 5,265 & 1,254 \\
\hline Processing industry & 1,276 & 1,613 & 2,281 & 3,005 & 3,352 & 4,295 & 1,978 \\
\hline Gas, electricity and water & 229 & 456 & 308 & 523 & 499 & 504 & 399 \\
\hline Construction & 289 & 409 & 433 & 584 & 665 & 905 & 467 \\
\hline Repairs, personal services, and trade & 941 & 1,885 & 2,003 & 2,287 & 2,488 & 3,028 & 1,526 \\
\hline Transport, warehouses, and communication & 671 & 1,013 & 1,527 & 1,974 & 2,254 & 2,863 & 1,721 \\
\hline Other & 1,962 & 2,557 & 3,400 & 4,129 & 5,689 & 6,496 & 3,702 \\
\hline \multicolumn{8}{|l|}{ Of which: } \\
\hline Financial services & 676 & 1,009 & 844 & 863 & 979 & 1,272 & 689 \\
\hline Education & 562 & 643 & 826 & 965 & 1,231 & 1,491 & 873 \\
\hline Public health and social assistance & 333 & 319 & 382 & 484 & 825 & 989 & 480 \\
\hline Other & 391 & 586 & 1,348 & 1,817 & 2,654 & 2,744 & 1,660 \\
\hline Net taxes & 1,403 & 1,323 & 1,998 & 2,278 & 2,867 & 3,941 & 1,948 \\
\hline GDP & 9,122 & 12,322 & 16,020 & 19,052 & 22,556 & 27,297 & 12,995 \\
\hline
\end{tabular}

Sources: Moldovan Department for Statistics and Sociology. 


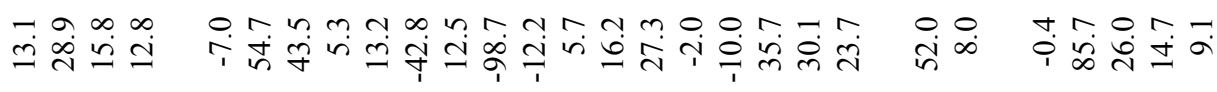

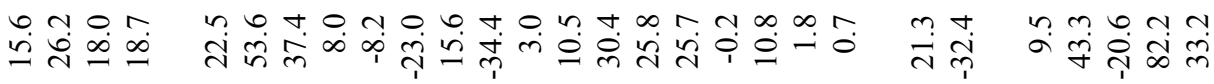

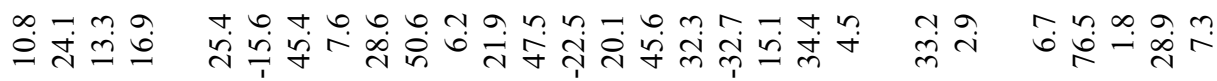

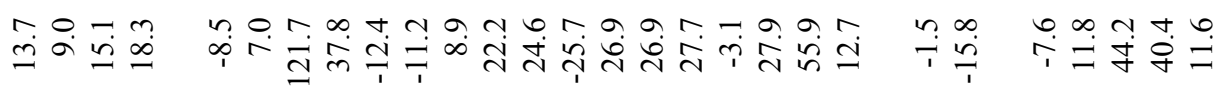

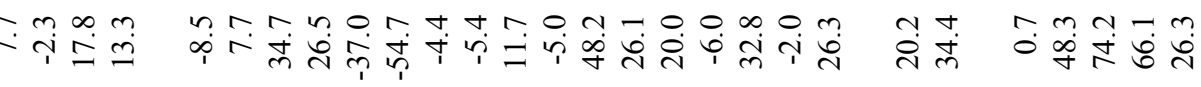

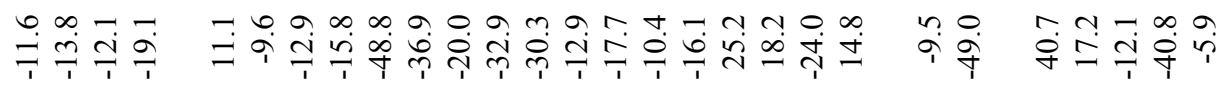

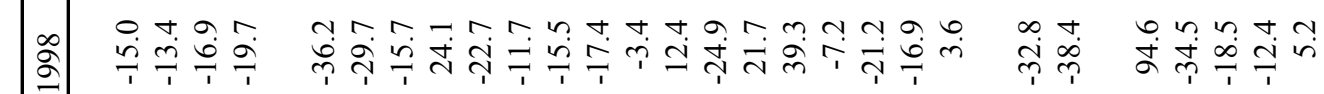

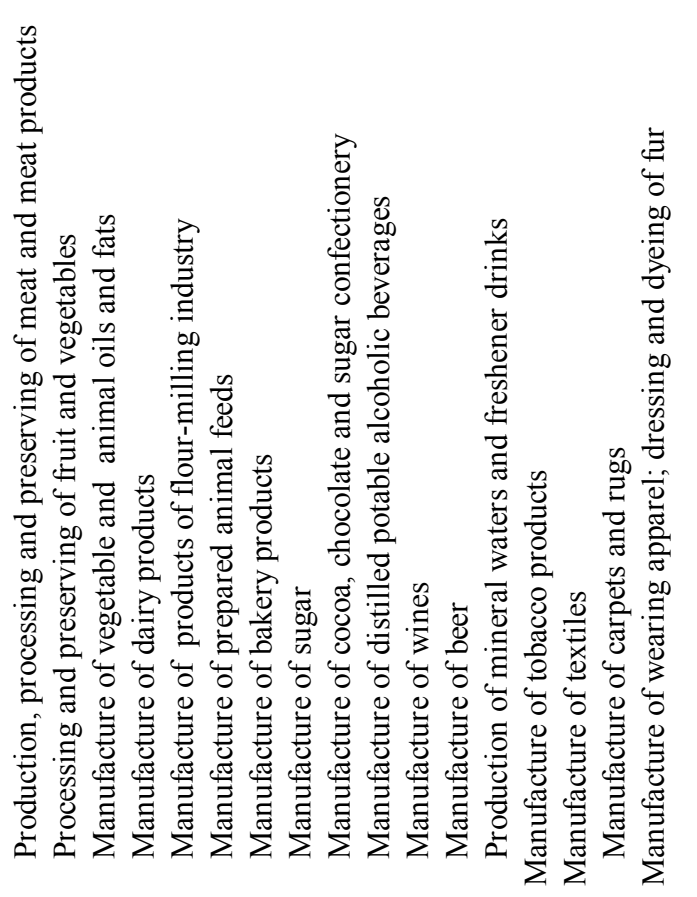




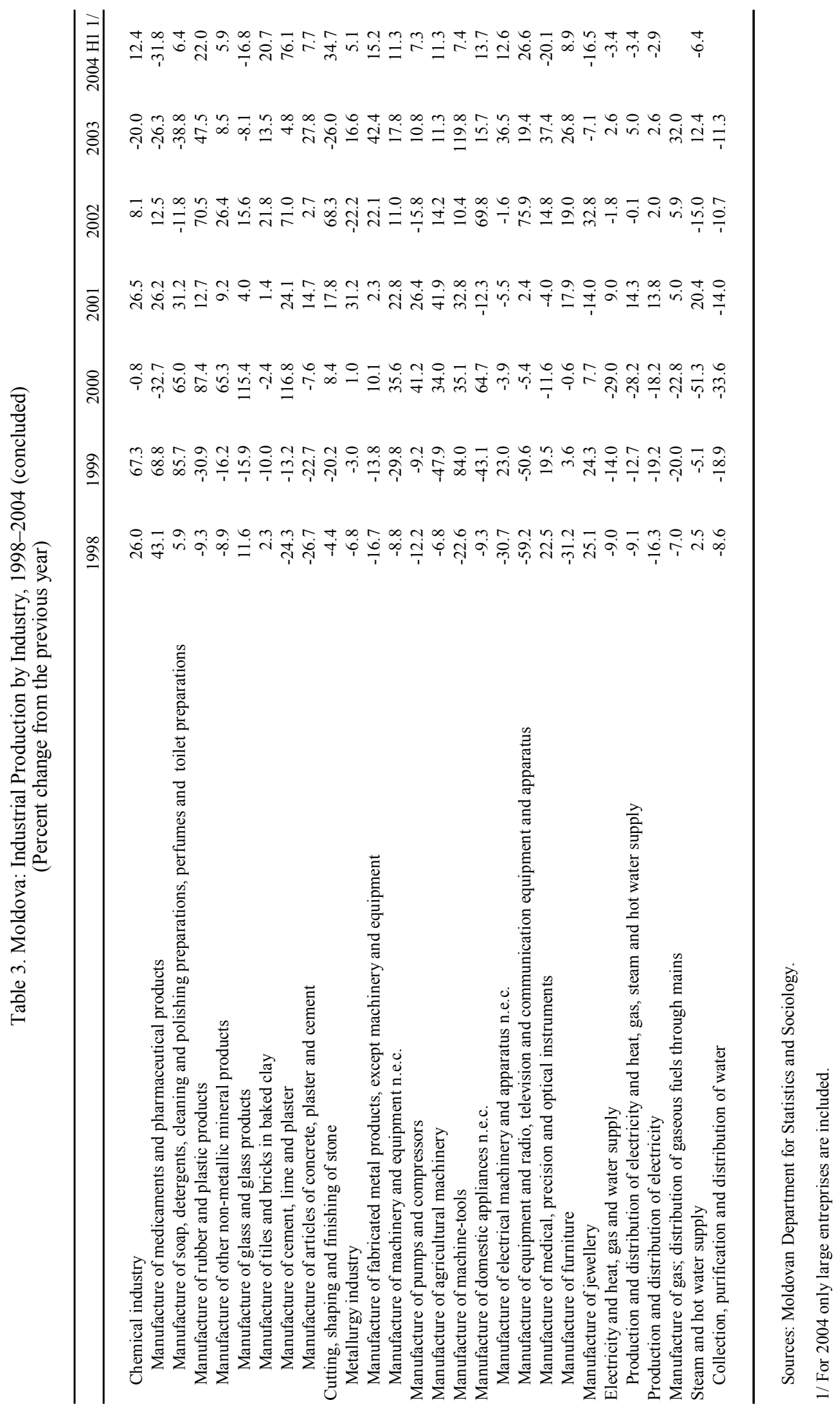


Table 4. Moldova: Agricultural and Industrial Production Indices, 1998-2004 (Percent change from same period of the previous year; period averages)

\begin{tabular}{|c|c|c|}
\hline & Agricultural Production 1/ & Industrial Production 2/ \\
\hline 1998 & -11.6 & -15.0 \\
\hline 1999 & -8.4 & -11.6 \\
\hline 2000 & -3.3 & 7.7 \\
\hline 2001 & 6.4 & 13.7 \\
\hline \multirow{2}{*}{\multicolumn{3}{|c|}{2003}} \\
\hline & & \\
\hline \multicolumn{3}{|l|}{$2004 \mathrm{H} 1$} \\
\hline \multicolumn{3}{|l|}{1998} \\
\hline I & -4.0 & 2.3 \\
\hline II & -2.0 & -1.3 \\
\hline III & -3.0 & -23.1 \\
\hline IV & -11.6 & -30.8 \\
\hline \multicolumn{3}{|l|}{1999} \\
\hline I & 0.8 & -29.9 \\
\hline II & -2.0 & -27.3 \\
\hline III & -7.0 & 1.9 \\
\hline IV & -8.4 & 0.6 \\
\hline \multicolumn{3}{|l|}{2000} \\
\hline I & -8.9 & 8.2 \\
\hline II & -9.7 & 9.3 \\
\hline III & -8.6 & 2.6 \\
\hline IV & -3.3 & 7.8 \\
\hline \multicolumn{3}{|l|}{2001} \\
\hline I & -8.0 & 5.2 \\
\hline II & -5.9 & 20.0 \\
\hline III & -1.7 & 12.4 \\
\hline IV & 6.4 & 14.1 \\
\hline \multicolumn{3}{|l|}{2002} \\
\hline I & 2.7 & 8.8 \\
\hline II & 4.8 & 13.2 \\
\hline III & 2.1 & 13.4 \\
\hline IV & 3.4 & 8.3 \\
\hline \multicolumn{3}{|l|}{2003} \\
\hline I & 1.6 & 12.8 \\
\hline II & -6.8 & 23.6 \\
\hline III & -19.9 & 21.5 \\
\hline IV & -13.6 & 2.3 \\
\hline 2004 I & -7.2 & 16.7 \\
\hline II & 0.9 & 9.7 \\
\hline
\end{tabular}

Sources: Moldovan Department for Statistics and Sociology.

$1 /$ Cummulative per end-quarter.

2/ Industrial production for 2000 and 2001 includes only medium and large enterprises. 


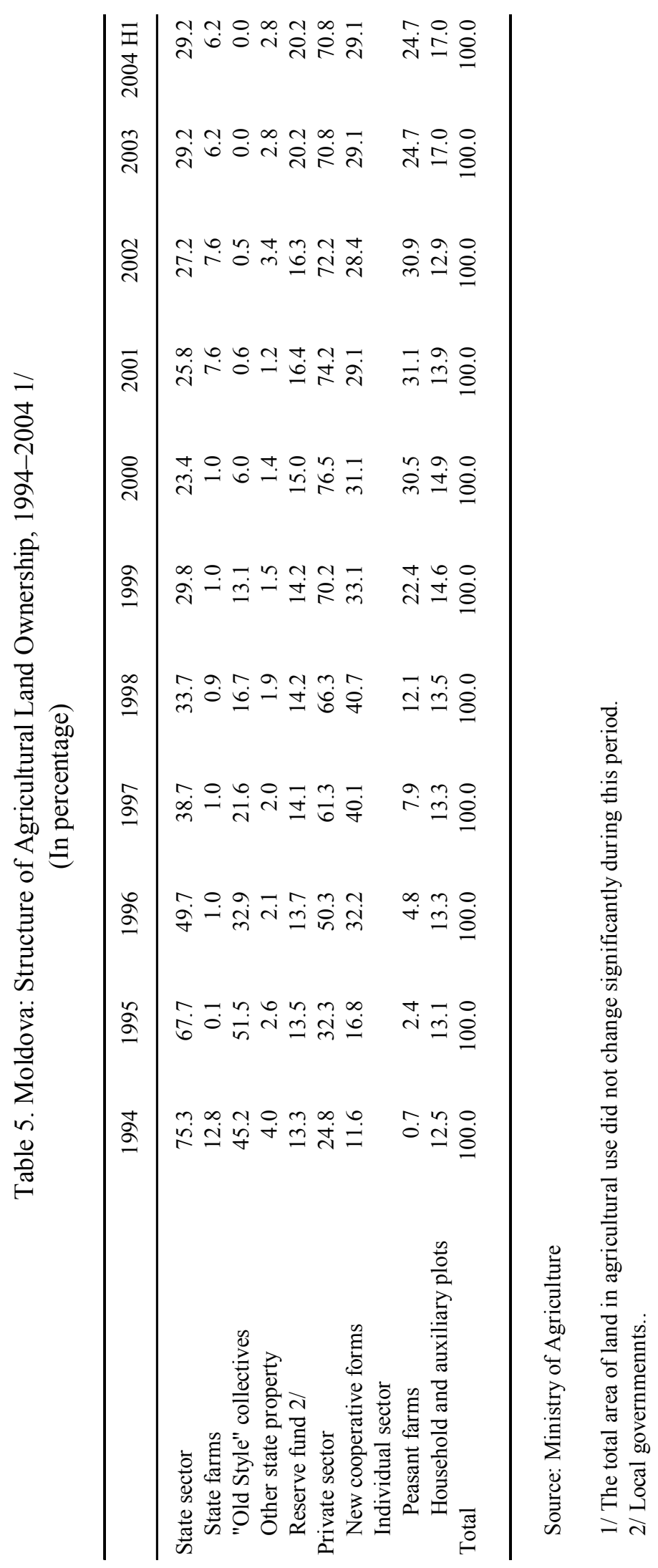


Table 6. Moldova: Agricultural Production by Sector, 1998-2004 (In millions of lei, constant 2000 prices)

\begin{tabular}{lrrrrrrr}
\hline & 1998 & 1999 & 2000 & 2001 & 2002 & 2003 & $2004 \mathrm{H} 1$ \\
\hline & & & & & & & \\
Total & 8,935 & 8,184 & 7,917 & 8,428 & 8,717 & 7,535 & 1,348 \\
Of which: & & & & & & \\
$\quad$ Agricultural enterprises & 3,770 & 2,574 & 2,132 & 2,397 & 2,525 & 1,864 & 337 \\
Individual sector & 5,165 & 5,611 & 5,785 & 6,031 & 6,193 & 5,671 & 1,101 \\
& & & & & & & \\
\hline
\end{tabular}

Source: Ministry of Agriculture. 
Table 7. Moldova: Agricultural Productivity of Public Versus Private Sector, 1998-2003 1/

\begin{tabular}{|c|c|c|c|c|c|c|}
\hline & 1998 & 1999 & 2000 & 2001 & 2002 & 2003 \\
\hline \multicolumn{7}{|c|}{ Share of land } \\
\hline Public & 20 & 16 & 8 & 8 & 7 & 12 \\
\hline Private & 66 & 70 & 76 & 76 & 77 & 87 \\
\hline \multicolumn{7}{|c|}{ Share of output } \\
\hline Public & 9 & 4 & 4 & 2 & 2 & 1 \\
\hline Private & 91 & 96 & 96 & 98 & 98 & 99 \\
\hline \multicolumn{7}{|c|}{ Share of output over share of land } \\
\hline Public & 0.4 & 0.3 & 0.5 & 0.2 & 0.3 & 0.1 \\
\hline Private & 1.4 & 1.4 & 1.3 & 1.3 & 1.3 & 1.1 \\
\hline
\end{tabular}

Source: Ministry of Agriculture

1/ Defined as the ratio between shares in agricultural output and share in land use; public sector includes state and "old" collective farms; and private sector includes "new" collectives and individual sector. 
Table 8. Moldova: Nominal Wages by Sector, 1998-2004 (In lei per month; period average)

\begin{tabular}{|c|c|c|c|c|c|c|c|}
\hline & 1998 & 1999 & 2000 & 2001 & 2002 & 2003 & $2004 \mathrm{H} 1$ \\
\hline Total & 250 & 305 & 408 & 544 & 692 & 891 & 1,025 \\
\hline Agriculture, forestry, hunting & 141 & 173 & 252 & 315 & 394 & 499 & 464 \\
\hline Fishery & 202 & 286 & 339 & 388 & 455 & 586 & 751 \\
\hline Industry & 424 & 519 & 683 & 827 & 1,002 & 1,271 & 1,425 \\
\hline Construction & 362 & 426 & 540 & 683 & 838 & 1,194 & 1,413 \\
\hline Wholesale and retail trade & 266 & 319 & 395 & 531 & 642 & 795 & 914 \\
\hline Hotels and restaurants & 230 & 277 & 358 & 485 & 571 & 827 & 966 \\
\hline Transport, warehouses, communication & 377 & 455 & 635 & 861 & 1,055 & 1,454 & 1,626 \\
\hline Banking, credit and insurance & 1,135 & 1,673 & 2,353 & 2,278 & 2,564 & 2,926 & 3,197 \\
\hline Real estate activity & 336 & 436 & 554 & 728 & 890 & 1,133 & 1,282 \\
\hline Central government, defense, social insurance & 392 & 439 & 518 & 742 & 989 & 1,050 & 1,163 \\
\hline Education & 183 & 193 & 248 & 337 & 463 & 610 & 720 \\
\hline Health careand social services & 184 & 187 & 230 & 315 & 439 & 579 & 791 \\
\hline Other public, social, and personal services & 227 & 235 & 296 & 391 & 505 & 671 & 745 \\
\hline
\end{tabular}

Sources: Moldovan Department for Statistics and Sociology. 
Table 9. Moldova: Employment by Sector, 1998-2003

(In thousands)

\begin{tabular}{|c|c|c|c|c|c|c|}
\hline & 1998 & 1999 & 2000 & 2001 & 2002 & 2003 \\
\hline Agriculture, forestry, hunting & 337.2 & 250.7 & 184.9 & 165.7 & 157.7 & 137.4 \\
\hline Fishery & 0.7 & 0.7 & 0.6 & 0.6 & 0.6 & 0.5 \\
\hline Industry & 147.3 & 130.8 & 114.6 & 125.8 & 123.7 & 125.5 \\
\hline Construction & 33.9 & 27.0 & 22.0 & 18.8 & 17.3 & 15.8 \\
\hline Wholesale and retail trade & 39.1 & 32.3 & 29.3 & 35.0 & 33.3 & 34.8 \\
\hline Hotels and restaurants & 8.7 & 6.8 & 5.4 & 6.0 & 6.3 & 6.6 \\
\hline Transport, warehouses, communication & 56.0 & 49.8 & 47.3 & 47.6 & 47.8 & 48.5 \\
\hline Banking, credit and insurance & 7.7 & 6.9 & 6.0 & 8.0 & 8.5 & 9.3 \\
\hline Real estate activity & 28.2 & 27.3 & 25.5 & 26.2 & 26.4 & 26.2 \\
\hline Central government, defense, social insurance & 52.0 & 48.4 & 50.0 & 50.7 & 53.9 & 56.7 \\
\hline Education & 144.1 & 131.2 & 124.4 & 122.3 & 124.8 & 125.2 \\
\hline Health care and social services & 85.1 & 74.1 & 68.4 & 67.4 & 67.0 & 63.3 \\
\hline Other public, social, and personal services & 23.3 & 19.4 & 18.4 & 18.0 & 18.0 & 18.5 \\
\hline Total & 963.3 & 805.4 & 696.8 & 692.1 & 685.3 & 668.3 \\
\hline
\end{tabular}

Sources: Moldovan Department for Statistics and Sociology. 
Table 10. Moldova: Unemployment, Unpaid Leave, and Part-time Employment, 1998-2004 (In thousands; excludes Transnistria)

\begin{tabular}{|c|c|c|c|c|c|c|}
\hline $\begin{array}{l}\text { End of } \\
\text { Period }\end{array}$ & & Unemployed & $\begin{array}{l}\text { Of which } \\
\text { Receiving } \\
\text { Benefit }\end{array}$ & Vacancies & $\begin{array}{l}\text { Workers on } \\
\text { Unpaid Leave }\end{array}$ & $\begin{array}{l}\text { Workers on } \\
\text { Part-time } \\
\text { Employment }\end{array}$ \\
\hline \multirow[t]{4}{*}{1998} & I & 39.1 & 7.7 & 6.4 & 82.1 & 30.8 \\
\hline & II & 33.7 & 7.2 & 7.4 & 97.7 & 38.0 \\
\hline & III & 33.8 & 7.8 & 6.6 & 120.9 & 43.1 \\
\hline & IV & 32.0 & 8.1 & 6.4 & 146.4 & 41.0 \\
\hline \multirow[t]{4}{*}{1999} & I & 40.1 & 8.3 & 5.5 & 100.6 & 33.4 \\
\hline & II & 38.6 & 8.7 & 6.4 & 116.6 & 32.3 \\
\hline & III & 40.4 & 10.6 & 7.3 & 127.4 & 35.6 \\
\hline & IV & 34.9 & 11.4 & 6.7 & 139.1 & 28.0 \\
\hline \multirow[t]{4}{*}{2000} & I & 36.7 & 11.1 & 7.8 & 75.5 & 22.1 \\
\hline & II & 33.2 & 9.8 & 8.6 & 85.8 & 22.1 \\
\hline & III & 33.9 & 8.3 & 8.3 & 94.9 & 26.4 \\
\hline & IV & 28.9 & 6.6 & 8.2 & 99.8 & 22.4 \\
\hline \multirow[t]{4}{*}{2001} & I & 35.9 & 5.8 & 7.7 & 56.9 & 18.8 \\
\hline & II & 33.5 & 5.5 & 7.7 & 65.1 & 18.5 \\
\hline & III & 29.8 & 6.1 & 7.6 & 70.5 & 22.9 \\
\hline & IV & 27.6 & 5.5 & 7.3 & 83.3 & 20.3 \\
\hline \multirow[t]{4}{*}{2002} & I & 35.6 & 5.3 & 9.7 & 50.8 & 17.9 \\
\hline & II & 30.4 & 4.4 & 10.5 & 58.4 & 17.5 \\
\hline & III & 29.1 & 4.2 & 9.2 & 64.3 & 17.5 \\
\hline & IV & 24.0 & 3.5 & 8.9 & 72.8 & 18.0 \\
\hline \multirow[t]{4}{*}{2003} & I & 34.8 & 3.1 & 9.0 & 39.3 & 16.4 \\
\hline & II & 27.7 & 2.5 & 10.4 & 44.5 & 15.7 \\
\hline & III & 24.2 & 1.6 & 11.7 & 49.8 & 20.8 \\
\hline & IV & 19.7 & 1.9 & 11.0 & 54.9 & 18.7 \\
\hline \multirow[t]{2}{*}{2004} & I & 29.0 & 1.7 & 9.9 & 27.9 & 16.0 \\
\hline & II & 22.8 & 1.7 & 12.1 & 32.4 & 16.0 \\
\hline
\end{tabular}

Sources: Moldovan Department for Statistics and Sociology. 
Table 11. Moldova: Monthly Prices, 1998-2004

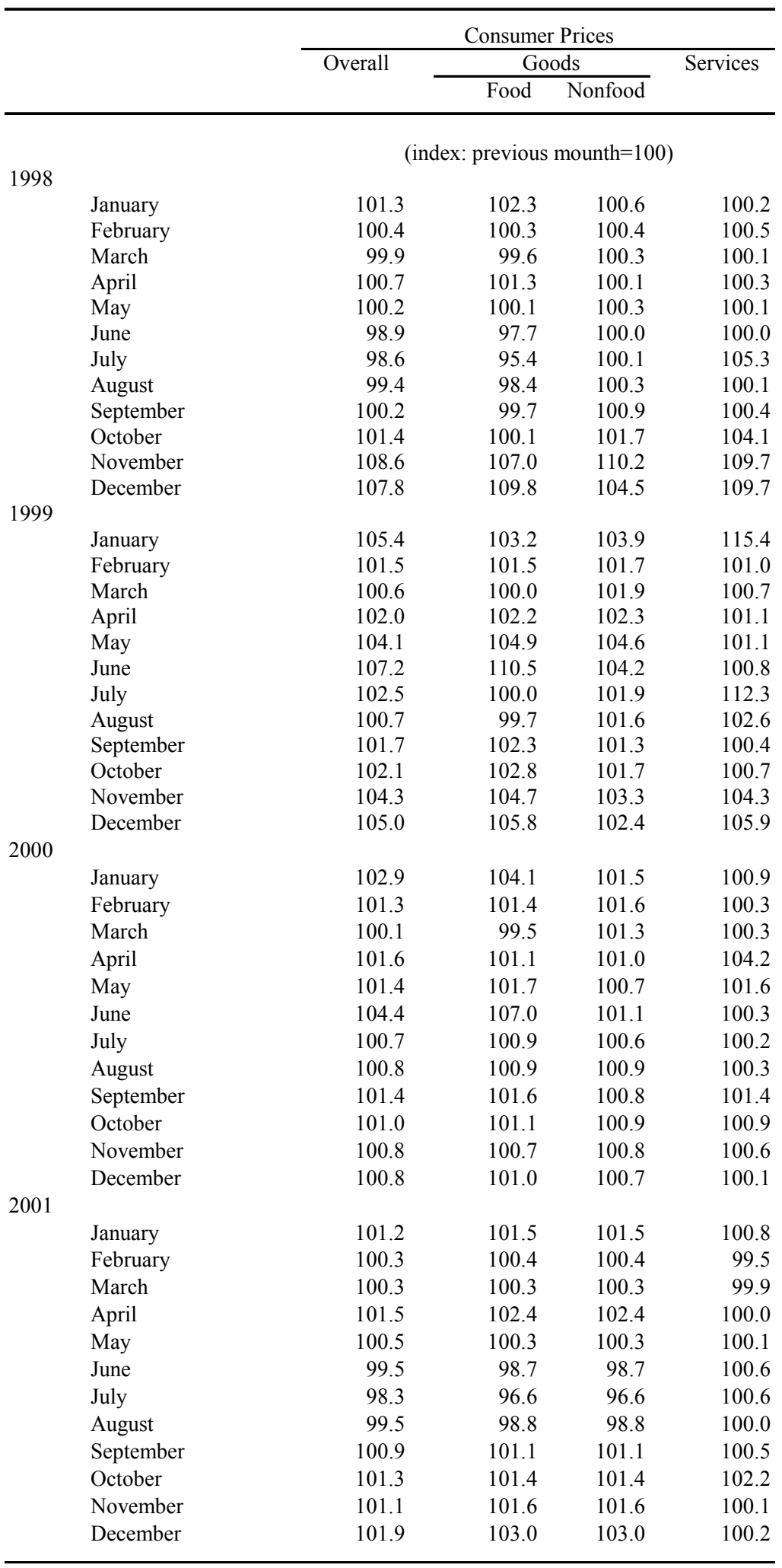


Table 11. Moldova: Monthly Prices, 1998-2004 (continued)

\begin{tabular}{|c|c|c|c|c|c|}
\hline & & \multicolumn{4}{|c|}{ Consumer Prices } \\
\hline & & \multirow[t]{2}{*}{ Overall } & \multicolumn{2}{|c|}{ Goods } & \multirow[t]{2}{*}{ Services } \\
\hline & & & Food & Nonfood & \\
\hline & & \multicolumn{4}{|c|}{ (index: previous mounth $=100$ ) } \\
\hline & January & 101.3 & 102.0 & 100.5 & 100.0 \\
\hline & February & 99.6 & 98.9 & 100.6 & 100.6 \\
\hline & March & 100.5 & 100.4 & 100.8 & 100.2 \\
\hline & April & 101.9 & 103.2 & 100.7 & 99.4 \\
\hline & May & 101.6 & 102.4 & 100.5 & 100.4 \\
\hline & June & 96.6 & 93.8 & 100.6 & 100.7 \\
\hline & July & 98.5 & 97.1 & 100.2 & 100.5 \\
\hline & August & 99.4 & 98.3 & 100.5 & 101.3 \\
\hline & September & 100.8 & 101.0 & 100.8 & 100.2 \\
\hline & October & 101.2 & 101.8 & 100.9 & 100.2 \\
\hline & November & 101.2 & 101.6 & 100.9 & 100.2 \\
\hline & December & 101.9 & 102.7 & 101.0 & 100.7 \\
\hline \multicolumn{6}{|l|}{2003} \\
\hline & January & 101.9 & 103.3 & 100.8 & 100.5 \\
\hline & February & 101.8 & 100.9 & 101.0 & 104.5 \\
\hline & March & 100.9 & 101.3 & 100.7 & 100.2 \\
\hline & April & 101.5 & 102.6 & 100.9 & 100.2 \\
\hline & May & 100.5 & 100.4 & 100.8 & 100.2 \\
\hline & June & 101.0 & 102.0 & 100.6 & 99.6 \\
\hline & July & 101.3 & 100.6 & 100.9 & 103.3 \\
\hline & August & 100.4 & 100.1 & 100.6 & 100.9 \\
\hline & September & 101.6 & 102.1 & 101.4 & 100.9 \\
\hline & October & 102.1 & 102.5 & 101.4 & 102.0 \\
\hline & November & 101.1 & 101.8 & 101.0 & 99.6 \\
\hline & December & 100.7 & 100.8 & 101.0 & 100.1 \\
\hline \multicolumn{6}{|l|}{2004} \\
\hline & January & 101.5 & 101.3 & 100.9 & 102.7 \\
\hline & February & 100.9 & 100.3 & 101.0 & 102.0 \\
\hline & March & 100.6 & 100.3 & 100.7 & 101.2 \\
\hline & April & 100.9 & 101.0 & 100.8 & 100.5 \\
\hline & May & 100.5 & 100.2 & 101.1 & 100.3 \\
\hline & June & 100.1 & 99.3 & 101.3 & 100.3 \\
\hline & July & 100.3 & 100.4 & 100.2 & 100.2 \\
\hline & August & 100.5 & 100.4 & 100.4 & 100.6 \\
\hline & September & 101.0 & 100.8 & 101.4 & 101.1 \\
\hline
\end{tabular}

Sources: Moldovan Department for Statistics and Sociology. 
Table 11. Moldova: Monthly Prices, 1998-2004 (concluded)

\begin{tabular}{|c|c|c|c|c|}
\hline & \multicolumn{4}{|c|}{ Consumer prices } \\
\hline & \multirow[t]{2}{*}{ Overall } & \multicolumn{2}{|c|}{ Goods } & \multirow[t]{2}{*}{ Services } \\
\hline & & Food & Nonfood & \\
\hline & \multicolumn{4}{|c|}{ (percentage change) } \\
\hline \multicolumn{5}{|c|}{ Annual averages } \\
\hline 1998 & 7.7 & 4.0 & 7.3 & 18.9 \\
\hline 1999 & 39.3 & 32.7 & 37.5 & 62.6 \\
\hline 2000 & 31.3 & 36.4 & 22.2 & 27.5 \\
\hline 2001 & 9.8 & 10.8 & 9.2 & 6.0 \\
\hline 2002 & 5.3 & 4.3 & 7.6 & 4.7 \\
\hline 2003 & 11.6 & 13.6 & 10.0 & 10.4 \\
\hline 2004H1 & 13.6 & 14.8 & 11.8 & 13.9 \\
\hline \multicolumn{5}{|c|}{ End-of-period } \\
\hline 1998 & 18.2 & 11.4 & 20.7 & 34.2 \\
\hline 1999 & 43.8 & 44.1 & 35.5 & 55.6 \\
\hline 2000 & 18.5 & 22.9 & 12.6 & 11.6 \\
\hline 2001 & 6.4 & 6.1 & 8.2 & 4.5 \\
\hline 2002 & 4.4 & 2.8 & 8.3 & 4.4 \\
\hline 2003 & 15.7 & 20.0 & 11.7 & 12.5 \\
\hline 2004H1 & 4.5 & 2.4 & 5.9 & 7.1 \\
\hline
\end{tabular}

Sources: Moldovan Department for Statistics and Sociology.and Fund staff estimates 
Table 12. Moldova: Exchange Rates, 1998-2004

\begin{tabular}{|c|c|c|c|c|c|}
\hline & & \multicolumn{2}{|c|}{ Leu/U.S. dollar } & \multicolumn{2}{|c|}{ Leu/1000 Russian Ruble } \\
\hline & & Average & $\overline{\mathrm{EoP}}$ & Average & $\overline{\text { EoP }}$ \\
\hline 1998 & & 5.38 & 8.32 & 0.63 & 0.40 \\
\hline 1999 & & 10.52 & 11.59 & 0.42 & 0.42 \\
\hline 2000 & & 12.43 & 12.38 & 0.44 & 0.43 \\
\hline 2001 & & 12.87 & 13.09 & 0.44 & 0.43 \\
\hline 2002 & & 13.57 & 13.82 & 0.43 & 0.43 \\
\hline 2003 & & 13.94 & 13.22 & 0.45 & 0.45 \\
\hline $2004 \mathrm{H} 1$ & & 12.43 & 11.95 & 0.43 & 0.41 \\
\hline \multirow[t]{12}{*}{1998} & January & 4.68 & 4.69 & 0.78 & 0.77 \\
\hline & February & 4.71 & 4.72 & 0.78 & 0.78 \\
\hline & March & 4.72 & 4.72 & 0.77 & 0.77 \\
\hline & April & 4.72 & 4.72 & 0.77 & 0.77 \\
\hline & May & 4.73 & 4.73 & 0.77 & 0.76 \\
\hline & June & 4.74 & 4.74 & 0.76 & 0.76 \\
\hline & July & 4.75 & 4.75 & 0.76 & 0.76 \\
\hline & August & 4.77 & 4.78 & 0.68 & 0.42 \\
\hline & September & 4.86 & 4.97 & 0.32 & 0.31 \\
\hline & October & 5.51 & 6.40 & 0.33 & 0.38 \\
\hline & November & 7.71 & 9.64 & 0.44 & 0.52 \\
\hline & December & 8.54 & 8.32 & 0.41 & 0.40 \\
\hline \multirow[t]{12}{*}{1999} & January & 8.53 & 8.75 & 0.37 & 0.38 \\
\hline & February & 8.74 & 8.73 & 0.38 & 0.38 \\
\hline & March & 8.95 & 9.68 & 0.37 & 0.39 \\
\hline & April & 9.60 & 9.95 & 0.37 & 0.40 \\
\hline & May & 10.87 & 11.61 & 0.44 & 0.47 \\
\hline & June & 11.74 & 11.46 & 0.48 & 0.47 \\
\hline & July & 11.09 & 10.99 & 0.45 & 0.45 \\
\hline & August & 10.98 & 11.02 & 0.44 & 0.44 \\
\hline & September & 10.98 & 10.96 & 0.43 & 0.43 \\
\hline & October & 10.99 & 11.20 & 0.42 & 0.43 \\
\hline & November & 12.00 & 12.04 & 0.45 & 0.45 \\
\hline & December & 11.73 & 11.59 & 0.44 & 0.42 \\
\hline \multirow[t]{12}{*}{2000} & January & 12.14 & 12.70 & 0.43 & 0.44 \\
\hline & February & 12.54 & 12.52 & 0.44 & 0.44 \\
\hline & March & 12.59 & 12.57 & 0.44 & 0.44 \\
\hline & April & 12.65 & 12.64 & 0.44 & 0.44 \\
\hline & May & 12.63 & 12.63 & 0.45 & 0.45 \\
\hline & June & 12.58 & 12.52 & 0.45 & 0.45 \\
\hline & July & 12.46 & 12.41 & 0.45 & 0.45 \\
\hline & August & 12.38 & 12.36 & 0.45 & 0.44 \\
\hline & September & 12.30 & 12.22 & 0.44 & 0.44 \\
\hline & October & 12.26 & 12.31 & 0.44 & 0.44 \\
\hline & November & 12.30 & 12.34 & 0.44 & 0.44 \\
\hline & December & 12.37 & 12.38 & 0.44 & 0.43 \\
\hline
\end{tabular}


Table 12. Moldova: Exchange Rates, 1998-2004 (concluded)

\begin{tabular}{|c|c|c|c|c|c|}
\hline & & \multicolumn{2}{|c|}{ Leu/U.S. dollar } & \multicolumn{2}{|c|}{ Leu/1000 Russian Ruble } \\
\hline & & Average & $\overline{\mathrm{EoP}}$ & Average & EoP \\
\hline \multirow[t]{12}{*}{2001} & January & 12.63 & 12.66 & 0.44 & 0.45 \\
\hline & February & 12.63 & 12.66 & 0.44 & 0.44 \\
\hline & March & 12.81 & 12.86 & 0.45 & 0.45 \\
\hline & April & 12.88 & 12.93 & 0.45 & 0.45 \\
\hline & May & 13.04 & 12.96 & 0.45 & 0.44 \\
\hline & June & 12.92 & 12.91 & 0.44 & 0.44 \\
\hline & July & 12.90 & 12.90 & 0.44 & 0.44 \\
\hline & August & 12.89 & 12.86 & 0.44 & 0.44 \\
\hline & September & 12.87 & 12.86 & 0.44 & 0.44 \\
\hline & October & 12.86 & 12.86 & 0.44 & 0.43 \\
\hline & November & 12.87 & 12.99 & 0.43 & 0.43 \\
\hline & December & 13.10 & 13.09 & 0.43 & 0.43 \\
\hline \multirow[t]{11}{*}{2002} & January & 13.09 & 13.09 & 0.43 & 0.43 \\
\hline & February & 13.11 & 13.20 & 0.43 & 0.43 \\
\hline & April & 13.49 & 13.51 & 0.43 & 0.43 \\
\hline & May & 13.57 & 13.68 & 0.43 & 0.44 \\
\hline & June & 13.79 & 13.87 & 0.44 & 0.44 \\
\hline & July & 13.72 & 13.62 & 0.43 & 0.43 \\
\hline & August & 13.60 & 13.59 & 0.43 & 0.43 \\
\hline & September & 13.58 & 13.57 & 0.43 & 0.43 \\
\hline & October & 13.63 & 13.79 & 0.43 & 0.43 \\
\hline & November & 13.89 & 13.94 & 0.44 & 0.44 \\
\hline & December & 13.94 & 13.82 & 0.44 & 0.43 \\
\hline \multirow[t]{12}{*}{2003} & January & 13.98 & 14.13 & 0.44 & 0.44 \\
\hline & February & 14.19 & 14.25 & 0.45 & 0.45 \\
\hline & March & 14.37 & 14.50 & 0.46 & 0.46 \\
\hline & April & 14.66 & 14.40 & 0.47 & 0.46 \\
\hline & May & 14.27 & 14.16 & 0.46 & 0.46 \\
\hline & June & 14.16 & 14.15 & 0.46 & 0.47 \\
\hline & July & 14.10 & 14.06 & 0.46 & 0.46 \\
\hline & August & 14.01 & 13.95 & 0.46 & 0.46 \\
\hline & September & 13.65 & 13.31 & 0.45 & 0.43 \\
\hline & October & 13.32 & 13.43 & 0.44 & 0.45 \\
\hline & November & 13.41 & 13.29 & 0.45 & 0.45 \\
\hline & December & 13.22 & 13.22 & 0.45 & 0.45 \\
\hline \multirow[t]{9}{*}{2004} & January & 13.20 & 13.14 & 0.46 & 0.46 \\
\hline & February & 12.91 & 12.71 & 0.45 & 0.45 \\
\hline & March & 12.63 & 12.41 & 0.44 & 0.44 \\
\hline & April & 12.09 & 11.80 & 0.42 & 0.41 \\
\hline & May & 11.78 & 12.01 & 0.41 & 0.41 \\
\hline & June & 11.98 & 11.95 & 0.41 & 0.41 \\
\hline & July & 11.96 & 11.96 & 0.41 & 0.41 \\
\hline & August & 12.02 & 12.07 & 0.41 & 0.41 \\
\hline & September & 12.12 & 12.16 & 0.41 & 0.42 \\
\hline
\end{tabular}

Source: National Bank of Moldova. 
Table 13. Moldova: General Government Operations, 1998-2003

(In millions of lei)

\begin{tabular}{|c|c|c|c|c|c|c|}
\hline & 1998 & 1999 & 2000 & 2001 & 2002 & 2003 \\
\hline Revenues and grants & 3,428 & 3,745 & 4,912 & 5,540 & 6,611 & 9,310 \\
\hline Revenues & 3,428 & 3,633 & 4,779 & 5,394 & 6,537 & 8,327 \\
\hline Tax revenues & 2,931 & 3,050 & 3,973 & 4,645 & 5,827 & 7,596 \\
\hline Profit tax & 179 & 233 & 275 & 350 & 428 & 577 \\
\hline Personal income tax & 224 & 219 & 265 & 348 & 468 & 623 \\
\hline VAT & 1,124 & 940 & 1,333 & 1,498 & 2,034 & 2,792 \\
\hline Excises & 375 & 445 & 658 & 681 & 658 & 888 \\
\hline Foreign trade taxes & 109 & 231 & 222 & 234 & 333 & 477 \\
\hline Other taxes & 137 & 199 & 227 & 231 & 263 & 259 \\
\hline Social Fund Revenues & 783 & 783 & 994 & 1,304 & 1,644 & 1,978 \\
\hline Health Fund Revenues & $\ldots$ & $\ldots$ & $\ldots$ & $\ldots$ & $\ldots$ & 2 \\
\hline Non-tax revenues & 497 & 583 & 806 & 749 & 710 & 731 \\
\hline NBM transfers & 187 & 160 & 387 & 230 & 139 & 221 \\
\hline Other & 310 & 423 & 419 & 519 & 571 & 510 \\
\hline Grants 1/ & 0 & 112 & 132 & 147 & 73 & 1 \\
\hline Revenues of extrabudetary and special funds & $\ldots$ & $\ldots$ & ... & $\ldots$ & $\ldots$ & 982 \\
\hline Expenditures and net lending (cash) & 4,099 & 4,507 & 5,420 & 5,589 & 7,057 & 9,129 \\
\hline (excl. project loan spending) & 3,806 & 4,122 & 5,169 & 5,399 & 6,723 & 8,791 \\
\hline National Economy & 281 & 287 & 331 & 298 & 417 & 999 \\
\hline Social sphere & 1,336 & 1,340 & 1,570 & 1,795 & 2,489 & 3,503 \\
\hline Education & 637 & 575 & 719 & 923 & 1,240 & 1,841 \\
\hline Health care & 393 & 335 & 464 & 542 & 792 & 1,068 \\
\hline Other & 306 & 430 & 387 & 329 & 457 & 594 \\
\hline Interest payments & 421 & 906 & 1,021 & 797 & 486 & 580 \\
\hline Capital expenditures & 206 & 103 & 175 & 206 & 258 & 0 \\
\hline Other expenditures & 621 & 642 & 776 & 926 & 1,251 & 1,898 \\
\hline Social Fund expenditures & 912 & 889 & 1,328 & 1,373 & 1,900 & 2,261 \\
\hline Health Fund expenditures & $\ldots$ & $\ldots$ & $\ldots$ & $\ldots$ & $\ldots$ & 12 \\
\hline Net lending & 30 & -44 & -32 & -17 & -78 & -52 \\
\hline Project loan spending & 293 & 385 & 252 & 190 & 334 & 338 \\
\hline Balance of extra-budgetary funds & 0 & 0 & 6 & 23 & 44 & 0 \\
\hline Overall balance (cash) & -671 & -762 & -503 & -25 & -402 & 181 \\
\hline Overall balance (commitments) & $-1,181$ & -745 & -226 & -54 & -200 & 60 \\
\hline
\end{tabular}

Sources: Ministry of Finance, Budget Department.

1/ Includes budget support grants only. 
Table 14. Moldova: General Government Operations, 1998-2003 (In percent of GDP)

\begin{tabular}{|c|c|c|c|c|c|c|}
\hline & 1998 & 1999 & 2000 & 2001 & 2002 & 2003 \\
\hline Revenues and grants & 37.6 & 30.4 & 30.7 & 29.1 & 30.0 & 34.1 \\
\hline Revenues & 37.6 & 29.5 & 29.8 & 28.3 & 29.7 & 30.5 \\
\hline Tax revenues & 32.1 & 24.8 & 24.8 & 24.4 & 26.4 & 27.8 \\
\hline Profit tax & 2.0 & 1.9 & 1.7 & 1.8 & 1.9 & 2.1 \\
\hline Personal income tax & 2.5 & 1.8 & 1.7 & 1.8 & 2.1 & 2.3 \\
\hline VAT & 12.3 & 7.6 & 8.3 & 7.9 & 9.2 & 10.2 \\
\hline Excises & 4.1 & 3.6 & 4.1 & 3.6 & 3.0 & 3.3 \\
\hline Foreign trade taxes & 1.2 & 1.9 & 1.4 & 1.2 & 1.5 & 1.7 \\
\hline Other taxes & 1.5 & 1.6 & 1.4 & 1.2 & 1.2 & 0.9 \\
\hline Social Fund Revenues & 8.6 & 6.4 & 6.2 & 6.8 & 7.5 & 7.2 \\
\hline Health Fund Revenues & $\ldots$ & $\ldots$ & $\ldots$ & $\ldots$ & $\ldots$ & 0.0 \\
\hline Non-tax revenues & 5.4 & 4.7 & 5.0 & 3.9 & 3.2 & 2.7 \\
\hline NBM transfers & 2.1 & 1.3 & 2.4 & 1.2 & 0.6 & 0.8 \\
\hline Other & 3.4 & 3.4 & 2.6 & 2.7 & 2.6 & 1.9 \\
\hline Grants 1/ & 0.0 & 0.9 & 0.8 & 0.8 & 0.3 & 0.0 \\
\hline Revenues of extrabudetary and special fi & $\ldots$ & $\ldots$ & $\ldots$ & $\ldots$ & $\ldots$ & 3.6 \\
\hline Expenditures and net lending (cash) & 44.9 & 36.6 & 33.8 & 29.3 & 32.0 & 33.4 \\
\hline (excl. project loan spending) & 41.7 & 33.5 & 32.3 & 28.3 & 30.5 & 32.2 \\
\hline National Economy & 3.1 & 2.3 & 2.1 & 1.6 & 1.9 & 3.7 \\
\hline Social sphere & 14.6 & 10.9 & 9.8 & 9.4 & 11.3 & 12.8 \\
\hline Education & 7.0 & 4.7 & 4.5 & 4.8 & 5.6 & 6.7 \\
\hline Health care & 4.3 & 2.7 & 2.9 & 2.8 & 3.6 & 3.9 \\
\hline Other & 3.4 & 3.5 & 2.4 & 1.7 & 2.1 & 2.2 \\
\hline Interest payments & 4.6 & 7.4 & 6.4 & 4.2 & 2.2 & 2.1 \\
\hline Capital expenditures & 2.3 & 0.8 & 1.1 & 1.1 & 1.2 & 0.0 \\
\hline Other expenditures & 6.8 & 5.2 & 4.8 & 4.9 & 5.7 & 7.0 \\
\hline Social Fund expenditures & 10.0 & 7.2 & 8.3 & 7.2 & 8.6 & 8.3 \\
\hline Health Fund expenditures & $\ldots$ & $\ldots$ & $\ldots$ & $\ldots$ & $\ldots$ & 0.0 \\
\hline Net lending & 0.3 & -0.4 & -0.2 & -0.1 & -0.4 & -0.2 \\
\hline Project loan spending & 3.2 & 3.1 & 1.6 & 1.0 & 1.5 & 1.2 \\
\hline Balance of extra-budgetary funds & 0.0 & 0.0 & 0.0 & 0.1 & 0.2 & 0.0 \\
\hline Overall balance (cash) & -7.4 & -6.2 & -3.1 & -0.1 & -1.8 & 0.7 \\
\hline Overall balance (commitments) & -12.9 & -6.0 & -1.4 & -0.3 & -0.9 & 0.2 \\
\hline
\end{tabular}

Sources: Ministry of Finance, Budget Department.

$1 /$ Includes budget support grants only. 
Table 15. Moldova: Central Government Operations, 1998-2003

(In millions of lei)

\begin{tabular}{|c|c|c|c|c|c|c|}
\hline & 1998 & 1999 & 2000 & 2001 & 2002 & 2003 \\
\hline Revenues, grants, and transfers & 1,972 & 2,281 & 3,048 & 2,884 & 3,292 & 5417 \\
\hline Revenues & 1,972 & 2,169 & 2,916 & 2,744 & 3,223 & 4485 \\
\hline Tax revenues & 1,599 & 1,708 & 2,292 & 2,246 & 2,827 & 3977 \\
\hline Profit tax & 106 & 134 & 136 & 118 & 138 & 209 \\
\hline Personal income tax & 1 & 2 & 1 & 2 & 2 & 3 \\
\hline VAT & 1,005 & 882 & 1,244 & 1,197 & 1,688 & 2382 \\
\hline Excises & 375 & 445 & 659 & 681 & 654 & 883 \\
\hline Foreign trade taxes & 109 & 231 & 231 & 234 & 333 & 477 \\
\hline Other taxes & 4 & 15 & 22 & 14 & 12 & 23 \\
\hline Non-tax revenues & 373 & 461 & 624 & 498 & 396 & 508 \\
\hline NBM transfers & 188 & 160 & 387 & 230 & 139 & 221 \\
\hline Other & 185 & 302 & 237 & 268 & 257 & 287 \\
\hline Grants 1/ & 0 & 112 & 124 & 131 & 69 & 72 \\
\hline Transfers from local governments & 0 & 0 & 8 & 9 & 0 & 38 \\
\hline Revenues of extrabudetary and special funds & $\ldots$ & $\ldots$ & $\ldots$ & $\ldots$ & $\ldots$ & 822 \\
\hline Expenditures and net lending (cash) 2/ & 2,322 & 2,853 & 3,364 & 2,938 & 3,556 & 5398 \\
\hline Expenditures & 2,360 & 2,780 & 3,396 & 2,963 & 3,635 & 5449 \\
\hline Social sphere & 743 & 831 & 987 & 941 & 1,253 & 1944 \\
\hline Education & 203 & 175 & 189 & 224 & 287 & 669 \\
\hline Health care & 159 & 174 & 164 & 168 & 263 & 453 \\
\hline Of which: Transfers to Health Fund & $\ldots$ & $\ldots$ & $\ldots$ & $\ldots$ & $\ldots$ & 1 \\
\hline Other expenditures & 381 & 481 & 634 & 549 & 703 & 822 \\
\hline Of which: Transfers to Social Fund & 137 & 125 & 335 & 301 & 371 & 387 \\
\hline Interest payments & 421 & 867 & 1,021 & 797 & 486 & 580 \\
\hline Domestic & 245 & 438 & 585 & 345 & 141 & 323 \\
\hline Foreign & 177 & 429 & 436 & 453 & 345 & 257 \\
\hline Capital expenditures & 122 & 34 & 41 & 20 & 22 & 0 \\
\hline Other expenditures & 878 & 806 & 1,071 & 1,048 & 1,663 & 2925 \\
\hline Of which: transfers to local governments & 477 & 316 & 476 & 378 & 724 & 823 \\
\hline Net lending & -38 & 73 & -33 & -25 & -80 & -51 \\
\hline Overall balance (cash) & -350 & -572 & -315 & -54 & -264 & 19 \\
\hline Primary balance (cash) & 72 & 295 & 705 & 743 & 223 & 599 \\
\hline
\end{tabular}

Sources: Ministry of Finance, Budget Department.

1/ Includes budget support grants only.

2/ Excluding project loans. 
Table 16. Moldova: Local Government Operations, 1998-2003

(In millions of lei)

\begin{tabular}{|c|c|c|c|c|c|c|}
\hline & 1998 & 1999 & 2000 & 2001 & 2002 & 2003 \\
\hline Revenues, grants, and transfers & $1,149.0$ & 995.4 & $1,391.3$ & $1,739.7$ & $2,398.7$ & 2847.0 \\
\hline Revenues & 671.9 & 679.3 & 907.2 & $1,346.1$ & $1,670.7$ & 1863.2 \\
\hline Tax revenues & 550.1 & 559.4 & 708.1 & $1,095.1$ & $1,356.8$ & 1640.2 \\
\hline Profit tax & 72.3 & 99.8 & 139.6 & 231.6 & 289.7 & 368.9 \\
\hline Personal income tax & 223.3 & 217.2 & 263.9 & 346.0 & 465.2 & 620.4 \\
\hline VAT & 119.5 & 58.3 & 98.3 & 301.0 & 346.0 & 410.3 \\
\hline Excises & 0.0 & 0.0 & 0.0 & 0.0 & 4.0 & 5.0 \\
\hline Foreign trade taxes & 0.0 & 0.0 & 0.0 & 0.0 & 0.0 & 0.0 \\
\hline Other taxes & 135.0 & 184.1 & 206.3 & 216.5 & 251.9 & 235.6 \\
\hline Non-tax revenues & 121.8 & 119.9 & 199.1 & 251.0 & 313.9 & 223.0 \\
\hline NBM transfers & 0.0 & 0.0 & 0.0 & 0.0 & 0.0 & 0.0 \\
\hline Other & 121.8 & 119.9 & 199.1 & 251.0 & 313.9 & 223.0 \\
\hline Grants 1/ & 0.0 & 0.0 & 8.6 & 15.6 & 4.2 & 1.1 \\
\hline Transfers from the central government & 477.1 & 316.1 & 475.5 & 378.0 & 723.8 & 822.7 \\
\hline Revenues of extrabudetary and special funds & $\ldots$ & $\ldots$ & $\ldots$ & $\ldots$ & $\ldots$ & 160.0 \\
\hline Expenditures and net lending (cash) & $1,114.4$ & 960.2 & $1,388.0$ & $1,767.7$ & $2,360.3$ & 2788.6 \\
\hline Expenditures & $1,114.4$ & 960.2 & $1,388.0$ & $1,767.7$ & $2,361.1$ & 2789.8 \\
\hline Social sphere & 757.9 & 656.5 & 928.2 & $1,154.8$ & $1,607.3$ & 1957.5 \\
\hline Education & 437.6 & 399.2 & 530.1 & 699.8 & 952.9 & 1172.4 \\
\hline Health care & 233.8 & 183.4 & 308.1 & 374.3 & 529.6 & 626.3 \\
\hline Transfers to the Health Fund & $\ldots$ & $\ldots$ & $\ldots$ & $\ldots$ & $\ldots$ & 10.0 \\
\hline Other & 86.5 & 73.9 & 90.0 & 80.7 & 124.8 & 158.8 \\
\hline Interest payments & 0.0 & 0.0 & 0.0 & 0.0 & 0.0 & 0.0 \\
\hline Domestic & 0.0 & 0.0 & 0.0 & 0.0 & 0.0 & 0.0 \\
\hline Foreign & 0.0 & 0.0 & 0.0 & 0.0 & 0.0 & 0.0 \\
\hline Capital expenditures & 84.0 & 76.3 & 140.5 & 185.6 & 236.7 & 0.0 \\
\hline Other expenditures & 177.2 & 161.3 & 228.3 & 265.4 & 311.5 & 832.3 \\
\hline Transfers to the central government & 0.0 & 0.0 & 7.9 & 9.0 & 0.0 & 38.0 \\
\hline Net lending & 0.0 & 0.0 & 0.0 & 0.0 & -0.8 & -1.2 \\
\hline Overall balance (cash) & 34.6 & 35.2 & 3.3 & -28.0 & 38.4 & 58.4 \\
\hline
\end{tabular}

Sources: Ministry of Finance, Budget Department.

1/ Includes budget support grants only. 
Table 17. Moldova: Central Government Expenditures by Main Budget Categories, 1998-2003 (In millions of lei)

\begin{tabular}{|c|c|c|c|c|c|c|}
\hline & 1998 & 1999 & 2000 & 2001 & 2002 & 2003 \\
\hline Expenditures and net lending 1/ & 2,322 & 2,853 & 3,364 & 2,938 & 3,556 & 5,397 \\
\hline National Economy & 196 & 243 & 276 & 157 & 211 & 576 \\
\hline Agriculture, forestry, fishery, and water administration & 71 & 114 & 147 & 42 & 79 & 272 \\
\hline Environmental protection and hydrometeorology & 6 & 6 & 7 & 9 & 10 & 39 \\
\hline Industry and construction & 2 & 3 & 4 & 5 & 6 & 10 \\
\hline Transport, road administration, communications, and informatics & 70 & 60 & 79 & 78 & 91 & 101 \\
\hline Public utilities administration and exploitation of housing stock & 34 & 6 & 0 & 1 & 1 & 34 \\
\hline Complex for fuel energy & 4 & 32 & 13 & 4 & 2 & 6 \\
\hline Other services related to economic activities & 9 & 21 & 26 & 19 & 23 & 115 \\
\hline Social sphere & 743 & 831 & 987 & 941 & 1,253 & 2,010 \\
\hline Education & 203 & 175 & 189 & 224 & 287 & 669 \\
\hline Health care & 159 & 174 & 164 & 168 & 263 & 453 \\
\hline Transfers to Health Fund & & & & & & 1 \\
\hline Social assistance and support & 301 & 415 & 562 & 474 & 594 & 719 \\
\hline Transfers to Social Fund & 137 & 125 & 335 & 301 & 371 & 387 \\
\hline Scientific research & 25 & 27 & 28 & 32 & 44 & 66 \\
\hline Culture, Arts, Sports & 55 & 39 & 45 & 43 & 66 & 103 \\
\hline Interest payments & 421 & 867 & 1,021 & 797 & 486 & 580 \\
\hline Foreign & 177 & 429 & 436 & 453 & 345 & 257 \\
\hline Capital expenditures & 122 & 34 & 41 & 20 & 22 & 0 \\
\hline Other expenditures & 878 & 806 & 1,071 & 1,048 & 1,663 & 2,283 \\
\hline General state services & 84 & 138 & 176 & 205 & 257 & 408 \\
\hline Foreign activities & 37 & 57 & 68 & 78 & 101 & 175 \\
\hline National defence & 57 & 63 & 64 & 77 & 95 & 129 \\
\hline Judical authorities and Constitutional jurisdiction & 24 & 29 & 35 & 38 & 52 & 76 \\
\hline Maintaining public order and national security & 174 & 177 & 207 & 241 & 394 & 524 \\
\hline Other expenditures & 503 & 342 & 522 & 409 & 765 & 972 \\
\hline Net lending & -38 & 73 & -33 & -25 & -80 & -51 \\
\hline
\end{tabular}

Sources: Ministry of Finance, Budget Department.

1/ Cash, excluding project loan spending. 
Table 18. Moldova: Local Government Expenditures by Main Budget Categories, 1998-2003 (In millions of lei)

\begin{tabular}{|c|c|c|c|c|c|c|}
\hline & 1998 & 1999 & 2000 & 2001 & 2002 & 2003 \\
\hline Expenditures and net lending 1/ & 1,030 & 884 & 1,248 & 1,582 & 2,124 & 2789 \\
\hline National Economy & 95 & 66 & 91 & 162 & 206 & 462 \\
\hline Agriculture, forestry, fishery, and water administration & 22 & 24 & 19 & 23 & 30 & 28 \\
\hline Environmental protection and hydrometeorology & 0 & 0 & 0 & 0 & 0 & 0 \\
\hline Industry and construction & 0 & 1 & 3 & 3 & 5 & 6 \\
\hline Transport, road administration, communications, and informatics & 8 & 10 & 20 & 19 & 24 & 27 \\
\hline Public utilities administration and exploitation of housing stock & 63 & 31 & 48 & 115 & 144 & 319 \\
\hline Complex for fuel energy & 0 & 0 & 0 & 0 & 1 & 79 \\
\hline Other services related to economic activities & 1 & 1 & 1 & 1 & 2 & 3 \\
\hline Social sphere & 758 & 657 & 928 & 1,155 & 1,607 & 1957.1 \\
\hline Education & 438 & 399 & 530 & 700 & 953 & 1172 \\
\hline Health care & 234 & 183 & 308 & 374 & 530 & 626 \\
\hline Transfers to Health Fund & & & & & & 10 \\
\hline Social assistance and support & 60 & 51 & 53 & 31 & 52 & 62 \\
\hline Culture, Arts, Sports & 27 & 23 & 37 & 50 & 73 & 97.1 \\
\hline Interest payments & 0 & 0 & 0 & 0 & 0 & 0 \\
\hline Domestic & 0 & 0 & 0 & 0 & 0 & 0 \\
\hline Foreign & 0 & 0 & 0 & 0 & 0 & 0 \\
\hline Other expenditures & 177 & 161 & 228 & 265 & 312 & 370 \\
\hline General state services & 63 & 86 & 131 & 139 & 167 & 199 \\
\hline National defence & 0 & 0 & 0 & 0 & 1 & 2 \\
\hline Judical authorities and Constitutional jurisdiction & 0 & 0 & 0 & 0 & 0 & 0 \\
\hline Maintaining public order and national security & 30 & 33 & 49 & 60 & 91 & 90 \\
\hline Other expenditures & 85 & 43 & 48 & 66 & 53 & 79 \\
\hline Net lending & 0 & 0 & 0 & 0 & -1 & -1 \\
\hline
\end{tabular}

Sources: Ministry of Finance, Budget Department.

1/ Cash, excluding project loan spending. 


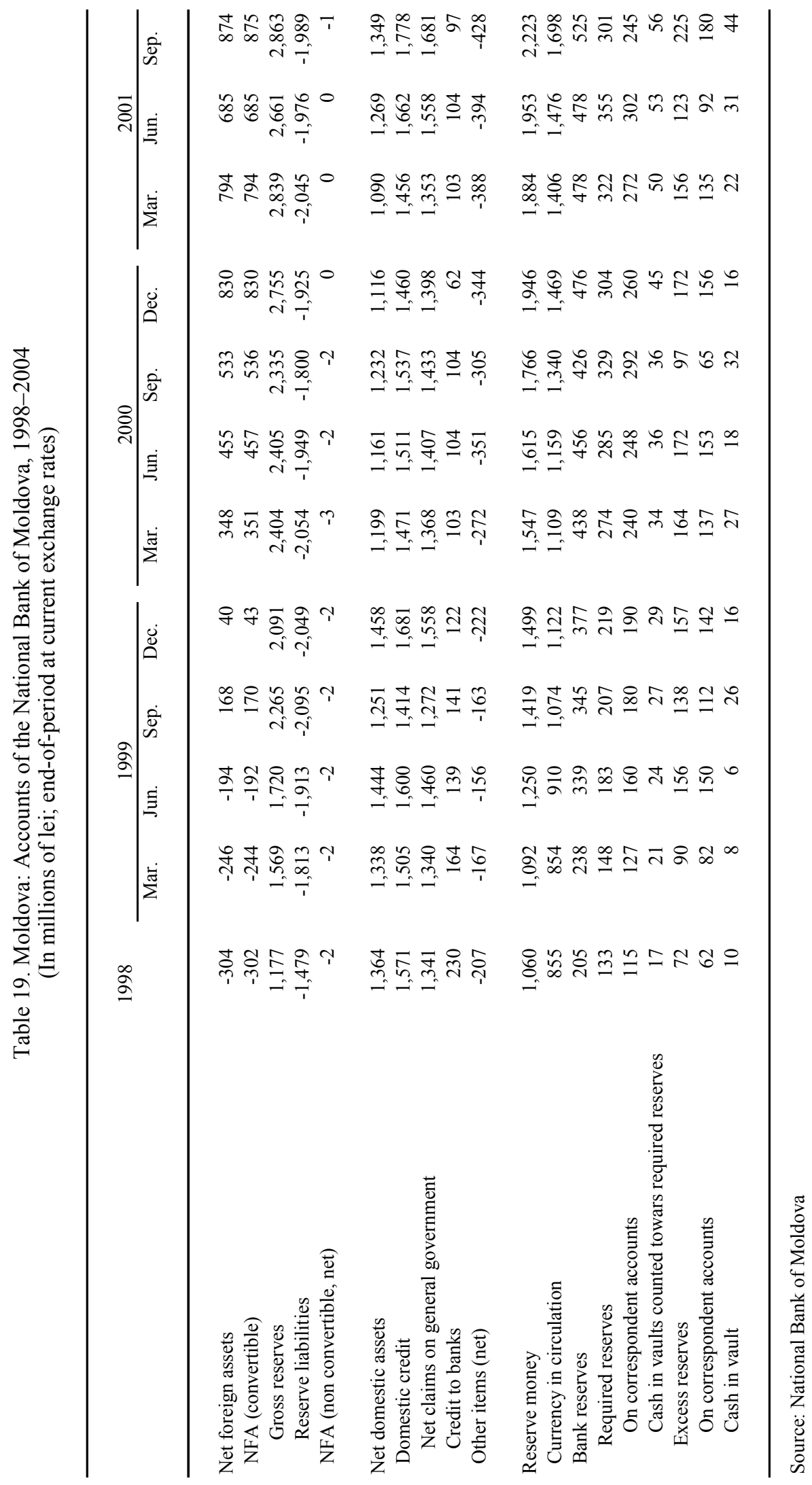




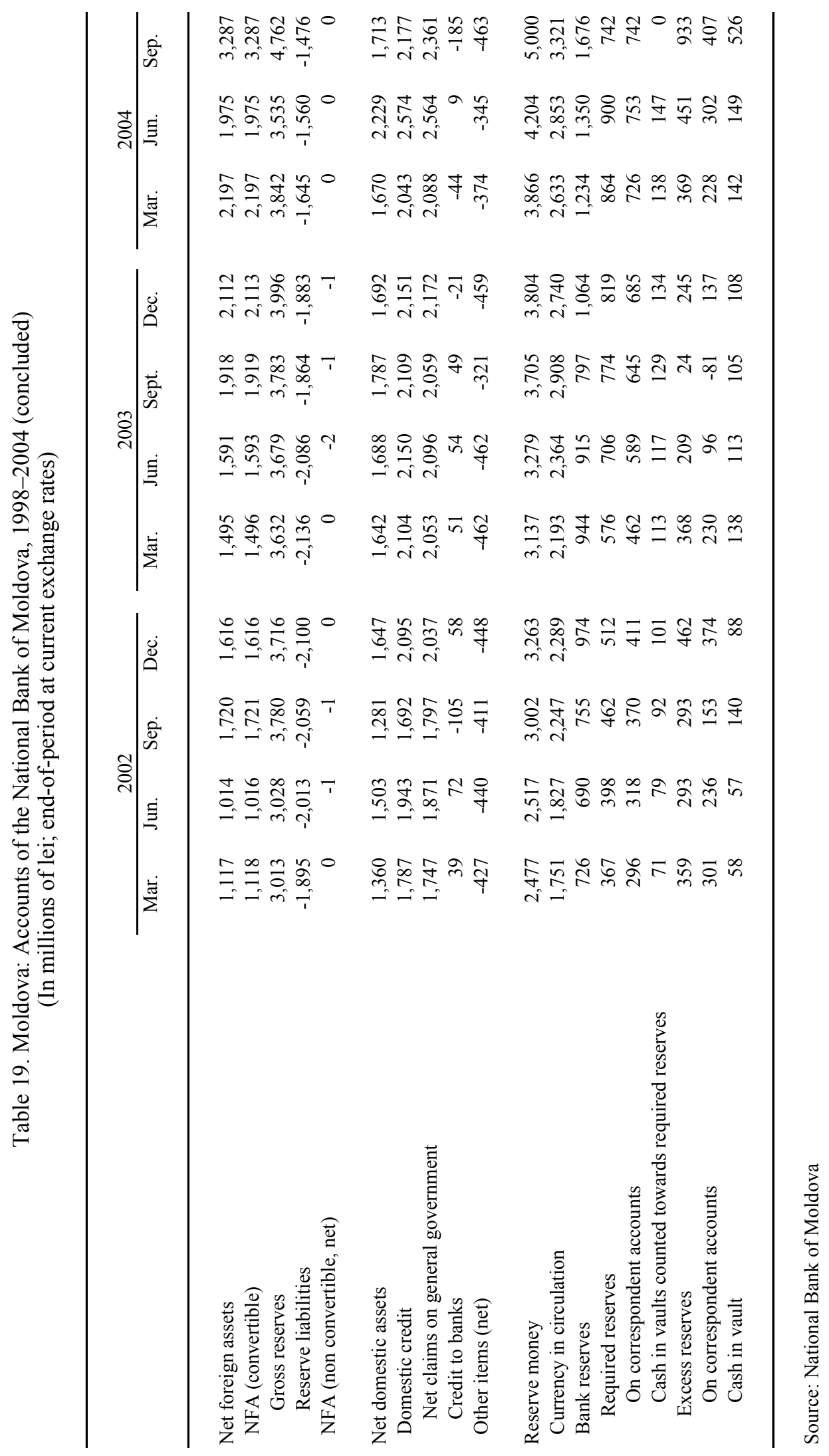




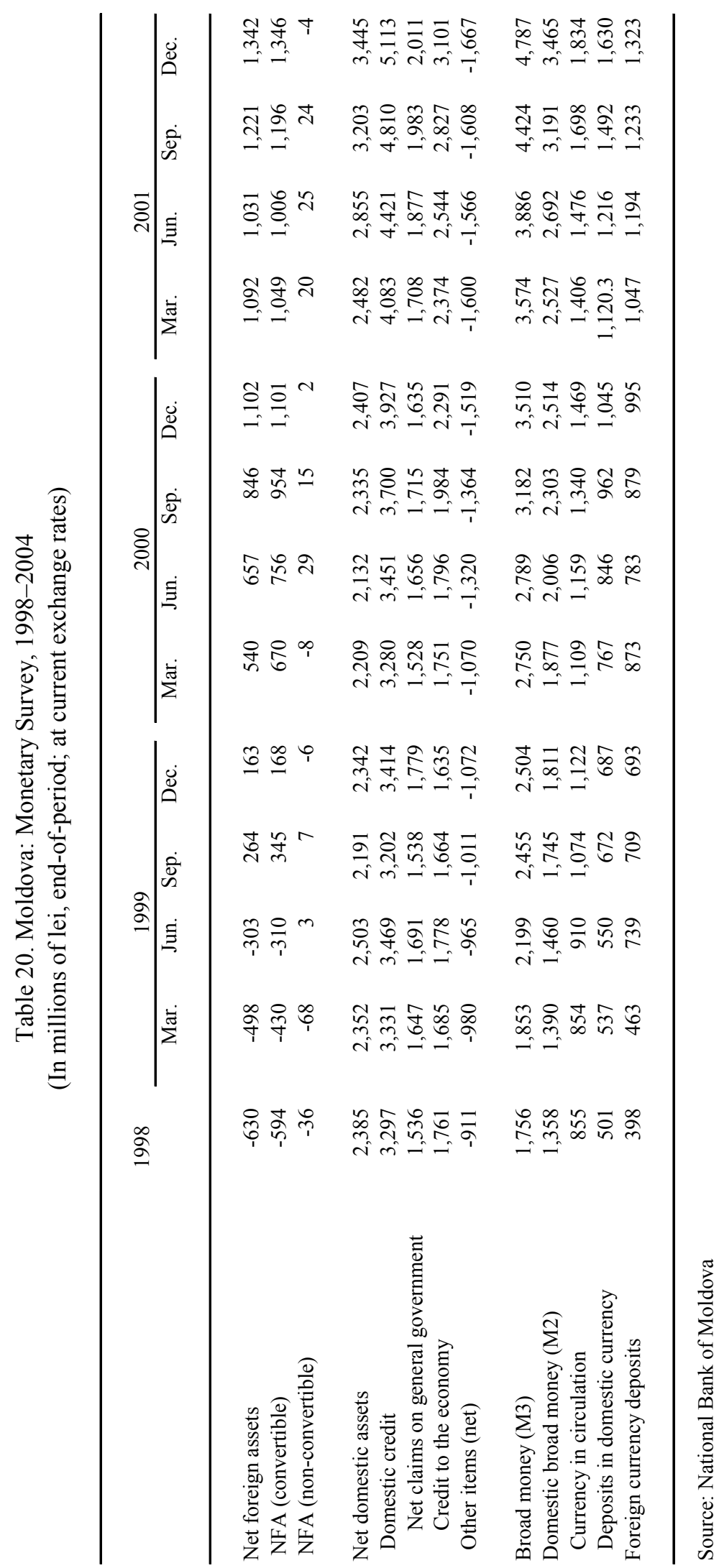




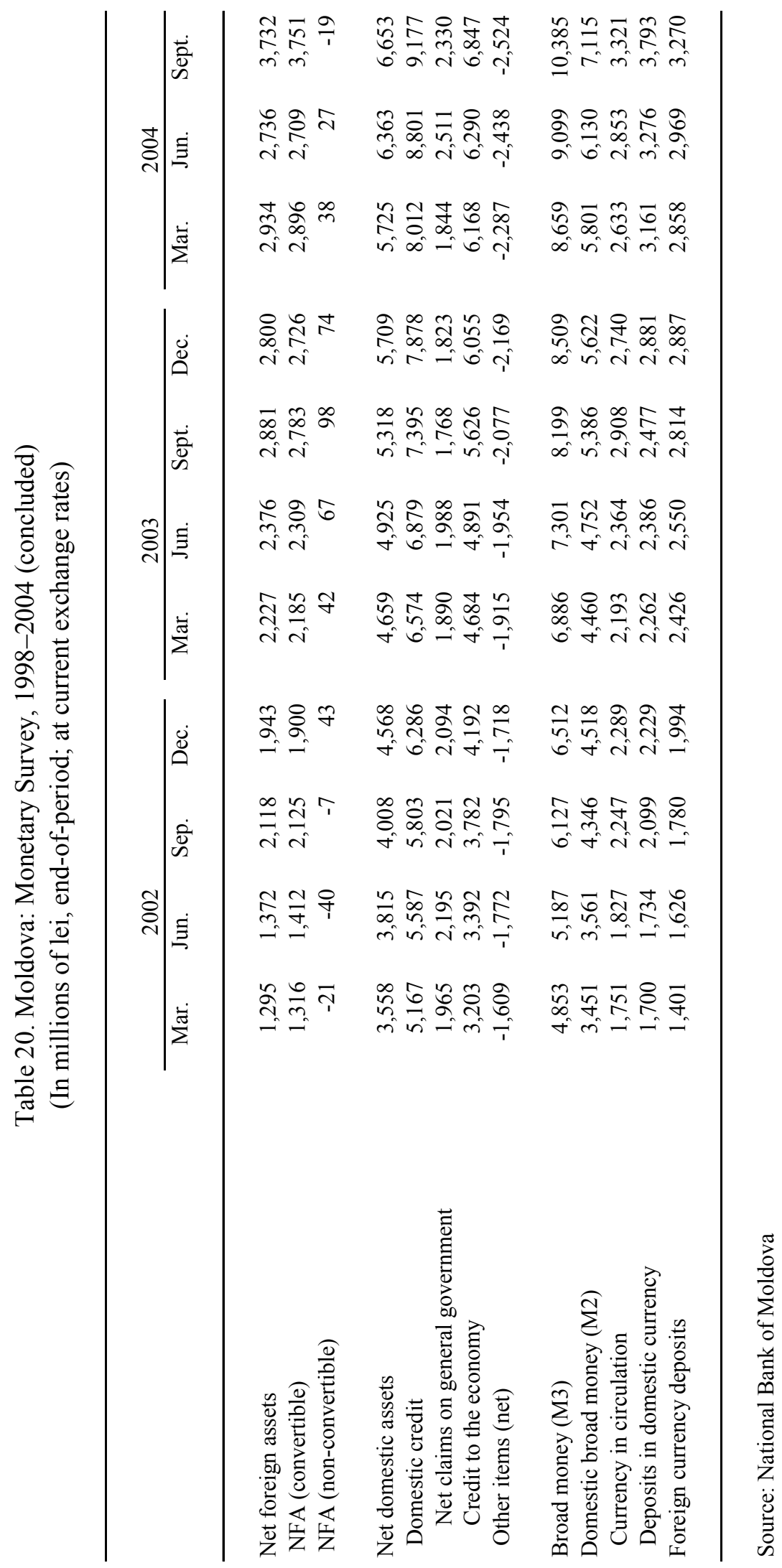


Table 21. Moldova: Balance of Payments, 1998-2004

(In millions of U.S. dollars; unless otherwise indicated)

\begin{tabular}{|c|c|c|c|c|c|c|c|}
\hline & 1998 & 1999 & 2000 & 2001 & 2002 & 2003 & $2004 \mathrm{H} 1$ \\
\hline Current account & -335 & -79 & -106 & -37 & -74 & -132 & -38 \\
\hline Merchandise trade balance & -388 & -137 & -294 & -313 & -378 & -622 & -294 \\
\hline Exports of goods & 644 & 474 & 477 & 567 & 660 & 806 & 466 \\
\hline Imports of goods & -1032 & -611 & -770 & -880 & -1038 & -1429 & -760 \\
\hline Of which: energy & -245 & -164 & -192 & -201 & -194 & -233 & -124 \\
\hline Balance of services & -47 & -42 & -37 & -38 & -40 & -46 & -48 \\
\hline Exports of services & 152 & 136 & 165 & 171 & 217 & 254 & 126 \\
\hline Imports of services & -199 & -178 & -202 & -209 & -257 & -300 & -174 \\
\hline Income (net) & 35 & 25 & 24 & 93 & 108 & 231 & 145 \\
\hline Compensation of employees & 100 & 86 & 96 & 123 & 189 & 294 & 178 \\
\hline Income on direct and portfolio investment & -35 & -23 & -34 & 15 & -39 & -28 & -15 \\
\hline Income on other investment & -30 & -38 & -38 & -45 & -41 & -34 & -18 \\
\hline Current transfers (net) & 65 & 76 & 201 & 220 & 236 & 304 & 158 \\
\hline Capital and financial account & 5 & -34 & 113 & -3 & 44 & 31 & 24 \\
\hline Capital account & 0 & 1 & -14 & -21 & -15 & -13 & -5 \\
\hline Financial account & 5 & -35 & 127 & 17 & 59 & 44 & 29 \\
\hline Direct investment & 76 & 38 & 127 & 53 & 130 & 68 & 35 \\
\hline Portfolio investment (net) & -59 & -7 & -4 & -7 & -28 & -23 & -7 \\
\hline Other investments & -12 & -65 & 4 & -28 & -43 & -1 & 1 \\
\hline Loans & 50 & 23 & 32 & 8 & -12 & -14 & 12 \\
\hline Government & 43 & -22 & -11 & -14 & -13 & -25 & -5 \\
\hline Disbursements & 96 & 42 & 21 & 15 & 20 & 23 & 10 \\
\hline Amortization & -53 & -64 & -32 & -30 & -33 & -48 & -15 \\
\hline Private net & 7 & 45 & 43 & 22 & 0 & 11 & 17 \\
\hline Other capital flows & -62 & -88 & -28 & -37 & -31 & 13 & -11 \\
\hline Errors and omissions & -23 & -4 & -10 & 14 & -18 & 77 & 42 \\
\hline Overall balance & -353 & -117 & -3 & -27 & -48 & -24 & 28 \\
\hline Financing & 353 & 117 & 3 & 27 & 48 & 24 & -28 \\
\hline Use of Fund credit & -64 & 5 & -12 & -2 & -6 & -22 & -11 \\
\hline Change of gross official reserves (increase -) & 226 & -49 & -47 & -9 & -27 & -14 & 5 \\
\hline Exceptional financing & 191 & 161 & 63 & 39 & 80 & 60 & -22 \\
\hline
\end{tabular}

Sources: National Bank of Moldova and Fund staff estimates. 
Table 22. Moldova: Composition of Trade, 1998-2004 (In percent of total)

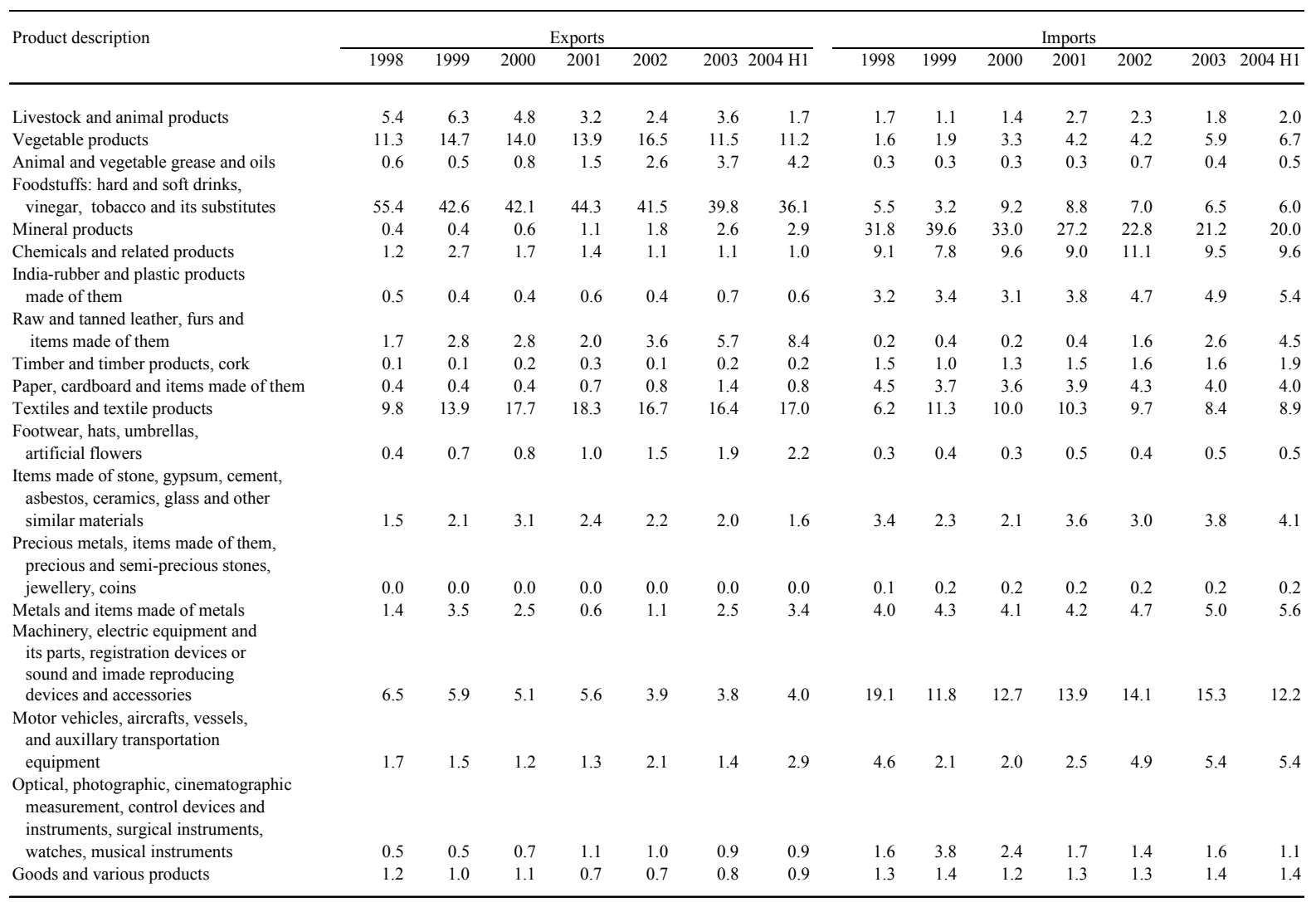

Sources: Moldovan Department for Statistics and Sociology. 


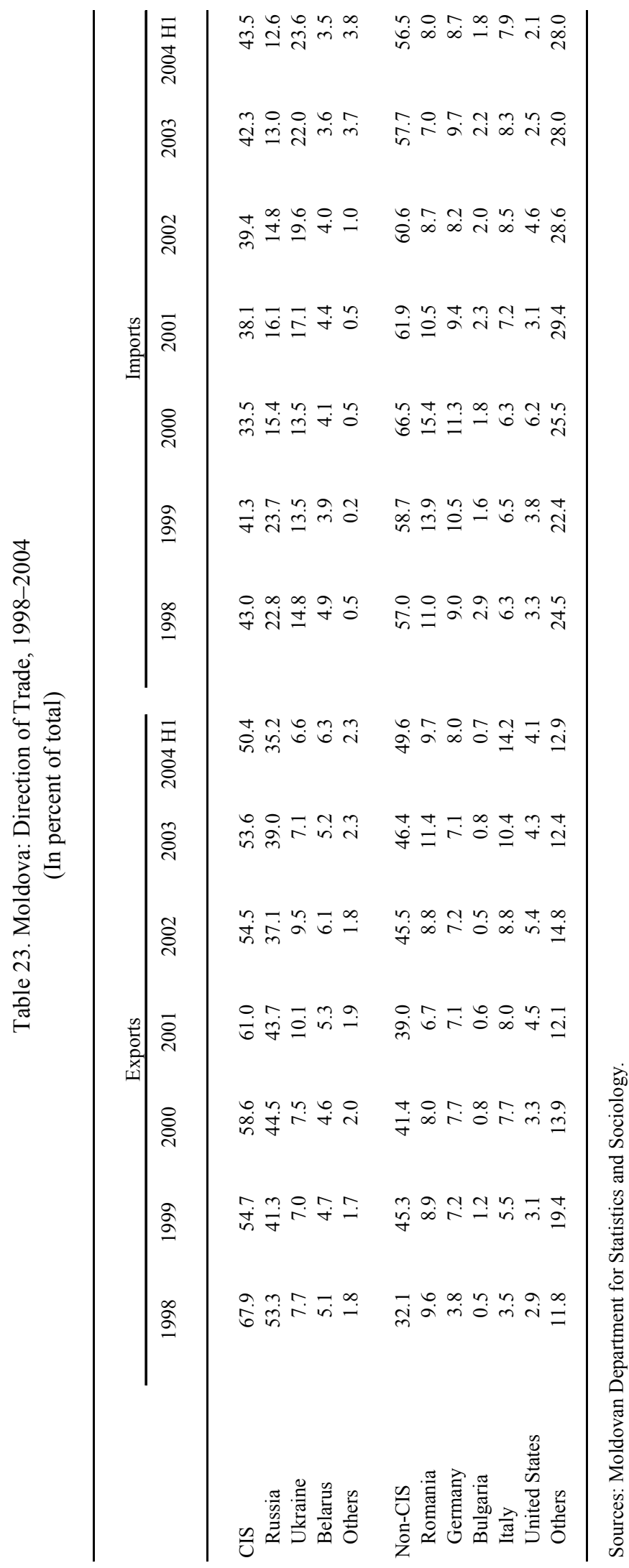


Table 24. Moldova: Import Tariff Developments, 1998-2003 1/

\begin{tabular}{|c|c|c|c|c|c|c|}
\hline & 1998 & 1999 & 2000 & 2001 & 2002 & 2003 \\
\hline Simple average tariff & 11.8 & 8.6 & 7.0 & 6.8 & 5.5 & 5.4 \\
\hline Weighted average tariff & 4.3 & 4.8 & 4.5 & 3.5 & 4.1 & 4.6 \\
\hline \multicolumn{7}{|c|}{ Distribution of imports by tariff band } \\
\hline 0 percent & 31 & 34 & 41 & 46 & 47 & 37 \\
\hline 5 percent & 29 & 28 & 30 & 29 & 29 & 30 \\
\hline 6.5 percent & 0 & 0 & 1 & 1 & 1 & 5 \\
\hline 8 percent & 0 & 0 & 0 & 0 & 0 & 1 \\
\hline 10 percent & 9 & 7 & 10 & 8 & 8 & 11 \\
\hline 15 percent & 1 & 31 & 16 & 15 & 15 & 16 \\
\hline 20 percent & 24 & 0 & 0 & 0 & 0 & 0 \\
\hline above 20 percent & 7 & 0 & 0 & 0 & 0 & 0 \\
\hline
\end{tabular}

Sources: Information provided by the Moldovan authorities and Fund staff estimates.

1/ Based on 4,015 import categories, excluding alcohol, tobacco, and vehicles. 
Table 25. Moldova: External Debt Indicators, 1998-2004

\begin{tabular}{lrrrrrrr}
\hline & 1998 & 1999 & 2000 & 2001 & 2002 & 2003 & 2004 H1 \\
& 1,453 & 1,493 & 1,593 & 1,563 & 1,681 & 1,752 & 1,650 \\
Total external debt & & & & & & & \\
& 1,001 & 935 & 1,045 & 979 & 1,009 & 1,030 & 901 \\
Public and publicly guaranteed debt & 514 & 586 & 565 & 552 & 589 & 600 & 593 \\
$\quad$ Multilateral creditors & 176 & 175 & 154 & 146 & 151 & 142 & 130 \\
$\quad$ IMF & 208 & 276 & 294 & 294 & 331 & 365 & 366 \\
$\quad$ World Bank & 59 & 75 & 66 & 70 & 69 & 60 & 63 \\
EBRD & 70 & 61 & 51 & 41 & 35 & 28 & 27 \\
EU & 0 & 0 & 0 & 1 & 3 & 5 & 7 \\
IFAD & 213 & 232 & 267 & 257 & 254 & 268 & 272 \\
Bilateral creditors & 34 & 36 & 29 & 24 & 24 & 26 & 26 \\
$\quad$ Japan & 71 & 74 & 122 & 122 & 122 & 125 & 129 \\
$\quad$ Russia & 62 & 61 & 60 & 59 & 56 & 57 & 57 \\
$\quad$ USA & 46 & 61 & 55 & 52 & 51 & 60 & 60 \\
$\quad$ Others & 274 & 116 & 213 & 170 & 166 & 162 & 36 \\
Commercial creditors & 117 & 143 & 231 & 297 & 371 & 421 & 454 \\
Private debt & 335 & 415 & 316 & 287 & 301 & 300 & 295 \\
Energy arrears & & & & & & &
\end{tabular}

Sources: National Bank of Moldova and Fund staff estimates. 